\title{
Utilización de Moodle en la gestión de información, documental y del conocimiento en grupos de investigación
}

\author{
Por Alejandro Uribe-Tirado, Liliana-María Melgar-Estrada y Jaime-Alberto Bornacelly-Castro
}

\begin{abstract}
Resumen: Se presenta la experiencia de dos grupos de investigación de la Escuela Interamericana de Bibliotecología de la Universidad de Antioquia (Medellín-Colombia), en cuanto a la adaptación y utilización de la plataforma Moodle (LMS) como un medio útil para la gestión documental, de información y del conocimiento en proyectos de investigación, más allá de su función original de gestión de cursos en línea. Esta experiencia, aún en proceso, ha posibilitado importantes logros en estos grupos de investigación, al convertirse en un modelo para esta universidad, y presentar esta opción de trabajo utilizando este tipo de plataforma, disponible en la mayoría de las universidades aunque inicialmente para uso de la docencia, como una herramienta clave para un adecuado desarrollo investigador en cualquier proyecto o grupo de investigación.
\end{abstract}

Palabras clave: Gestión documental, Gestión de información, Gestión del conocimiento, Aprendizaje colaborativo, Grupos de investigación, Sistemas de administración de aprendizaje, Aprendizaje virtual, Investigación virtual, Moodle.

Title: Moodle learning management system as a tool for information, documentation, and knowledge management by research groups

Abstract: Two research groups at the Inter-American School of Library Science, University of Antioquia (Medellin-Colombia), share their experience and lessons learned during the adaptation and use of the Moodle learning management system (LMS) platform as a useful means for information, documentation, and knowledge management in research projects. Moodle was developed as a tool for online course management. This ongoing adaptation has yielded important benefits to the researchers and become a model for the University. This type of technological platform, available to most universities for instructional purposes, has potential to become a key developmental tool for any research project or group.

Keywords: Document management, Information management, Knowledge management, Collaborative learning, Research groups, Learning system management, E-learning, E-research, Moodle.

Uribe-Tirado, Alejandro; Melgar-Estrada, Liliana-María; Bornacelly-Castro, Jaime-Alberto. "Utilización de Moodle en la gestión de información, documental y del conocimiento en grupos de investigación". En: El profesional de la información, 2007, septiembre-octubre, v. 16, n. 5, pp. 468-474.

DOI: $10.3145 /$ epi.2007.sep.09

\section{Introducción}

ESTE TRABAJO SE BASA EN LA EXPERIENCIA que han desarrollado durante 2006 y 2007 dos grupos de investigación universitarios al adaptar un software utilizado con fines de enseñanza a las necesidades de los grupos de investigación durante el desarrollo de proyectos.
Dichos proyectos de investigación son: "Acceso, conocimiento y uso de internet en la Universidad de Antioquia", perteneciente al Grupo de Investigación en Gestión del Conocimiento, y "Exploración de los discursos y las prácticas de la promoción de la lectura en las bibliotecas públicas de Medellín: una revisión del lugar social de la biblioteca pública en la formación de los lectores", del Grupo de Investigación en Biblioteca Pública, ambos de la Escuela Interamericana de Bibliotecología (EIB) de la Universidad de Antioquia (UA).

\section{La elección}

La idea de utilizar una plataforma tecnológica para apoyar las 
labores investigadoras de los dos proyectos mencionados surgió en el momento de iniciarlos, cuando se identificaron dos aspectos críticos para el adecuado desarrollo de los mismos. Por un lado, el hecho de ser grupos inter y multidisciplinares, con personas vinculadas a diferentes dependencias universitarias y a instituciones sociales y gubernamentales, hacía difíciles las reuniones de trabajo presenciales, por lo que se pensó en un mediador tecnológico que facilitara el trabajo colaborativo. Por otro lado, las lecciones aprendidas de anteriores investigaciones, en los que se observó que uno de los mayores problemas es que los procesos (de gestión, de aprendizaje, de formalización, etc.) no quedan registrados en ningún documento o espacio interactivo y de libre consulta al terminar los proyectos (como memoria para otros futuros). Los informes finales que se entregan a las instancias patrocinadoras y los artículos que se publican dan cuenta de los resultados pero rara vez de los procesos.

Al buscar soluciones a estas dos problemáticas se identificaron tres opciones:

- creación de una intranet,

- implementación de un sistema de gestión de contenidos $(C M S)^{1}$, elegido de entre los múltiples softwares libres o comerciales, $\mathrm{y}$

- adaptación de plataformas de e-learning ya existentes en el ámbito universitario.

Se escogió la última opción para así poder aprovechar la experiencia ya adquirida en la Universidad de Antioquia sobre el software $M_{o o d l e}{ }^{2}$, utilizado para la gestión de cursos en línea. Para esta universidad fue novedad su aplicación específica a proyectos de investigación, lo cual se ha convertido en un modelo para otros grupos y proyectos. Sabemos que lo mismo ha sucedido en otras universidades de diferentes países ${ }^{3}$.

\section{LMS y Moodle}

Los sistemas de administración del aprendizaje (learning management systems, LMS) comenzaron a aparecer como una etapa evolutiva del uso de herramientas tecnológicas basadas en internet para la educación, tras los cursos estáticos que presentaban el programa, algunos links o archivos con contenidos y la opción de interacción asincrónica a través del correo electrónico. Estos sistemas tuvieron su masificación en las universidades a finales del siglo pasado e inicios de éste. En las primeras experiencias predominó la utilización de software comercial, pero con el paso de los años y el fortalecimiento del software libre con comunidades virtuales de usuarios a nivel mundial, ha sido éste el que ha logrado una mayor aceptación, ya que al no tener que pagar licencias se libera dinero para otras inversiones. Ocurre incluso que el software libre evoluciona más rápidamente.

Moodle 4 se empezó a usar en la Universidad de Antioquia tras un estudio entre varios paquetes informáticos de e-learning 5 . Se caracteriza, como se indica en su sitio web oficial, por "permitir diseñar y publicar en la web materiales educativos, ofreciendo un sistema de comunicaciones con aplicaciones de correo electrónico, chat, foro, entre otros, y que, además, permite administrar los cursos y arroja estadísticas sobre el uso de éstos. (...) Su nombre son las siglas del inglés Modular object-oriented dynamic learning environment (Entorno de aprendizaje modular y dinámico orientado a objetos)".

\section{La implementación}

Decidida esta opción, se revisó su estructura y configuración, y se adaptó a la dinámica de los procesos de investigación, evidentemente diferente de la de una asignatura académica o un curso en línea. Se definió que cada etapa de las investigaciones serían los componentes de cada uno de los bloques de Moodle (figura 1) y que en cada uno de ellos se pensarían las posibilidades de interactividad (contenidos, multimedia, mapas conceptuales, etc.) y de interacción (foros, chat, wiki, etc.) que se requerían ${ }^{6}$.

Para una adecuada gestión de los contenidos y las actividades, se distribuyeron roles para la configuración inicial: reuniones y trabajo conjunto para el acuerdo entre los investigadores sobre la estructura que debía tener el sitio y las herramientas colaborativas que se utili-

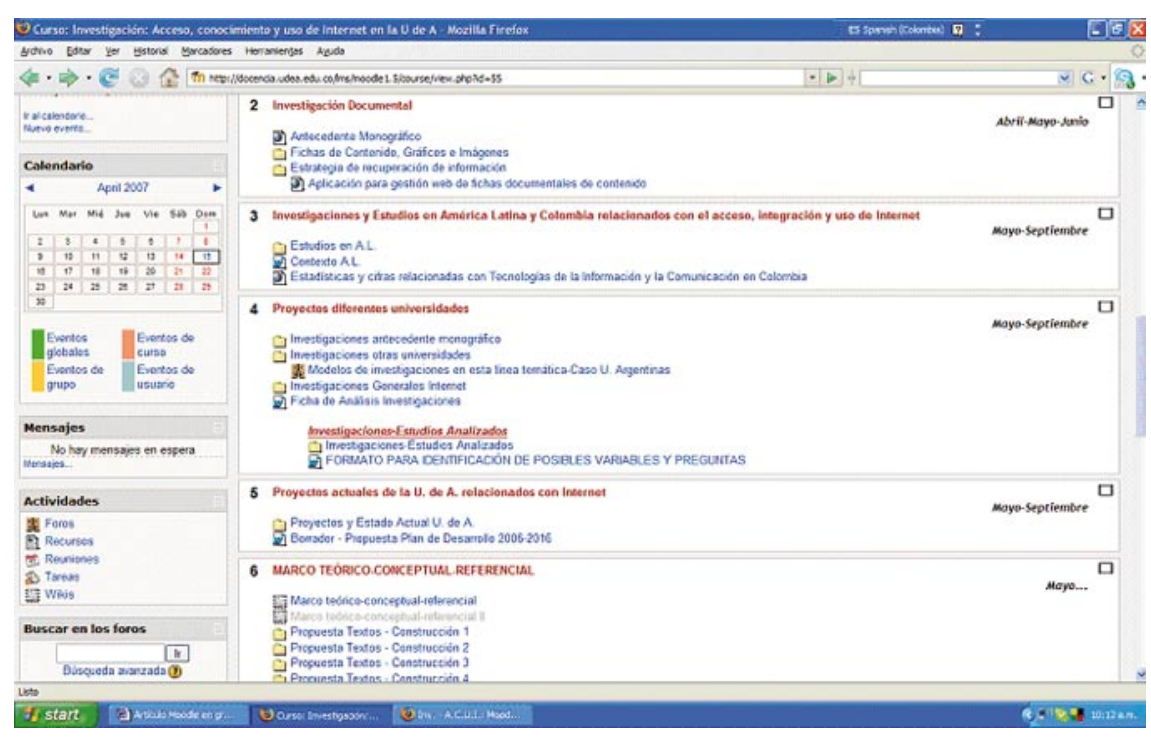

Figura 1. Esquema de bloques de Moodle utilizados por el grupo como recuento de fases de la investigación. Se observan algunas de las herramientas utilizadas 
zarían; asignación de un administrador encargado de su diseño inicial; y asignación posterior del perfil de administrador a todos los miembros de los grupos de investigación.

Se buscó así garantizar la construcción y utilización del sitio no sólo inicialmente, sino en todas las etapas posteriores a lo largo del desarrollo de la investigación. De este modo se constituyó un espacio de trabajo netamente colaborativo que se asumió como de responsabilidad de todos los investigadores. Es decir, éstos tenían tanto el rol de "productores" como de de "usuarios", lo cual permitía además lograr indirectamente un propósito básico entre el grupo: la alfabetización digital en la práctica y disminuir la brecha digital entre los integrantes del equipo investigador. Se tuvieron en cuenta las diferencias en habilidades entre ellos, y la primera realización interactiva en el uso de la plataforma fue el vídeo Guía para el uso de Moodle como espacio para un grupo de investigación (figura 2).

Finalmente, se determinó que este espacio sería de acceso restringido mientras se llevaban a cabo las investigaciones, emulando con ello una intranet, y que al finalizar los proyectos sería -junto con el infor- me final y los artículos- una fuente oficial de divulgación de la investigación, tanto de procesos como de resultados, totalmente en línea y a texto completo (open access-creative commons).

\section{Gestión de información, de documentos y del conocimiento}

Los grupos de investigación universitarios, concebidos como organizaciones para la generación de nuevo conocimiento, realizan prácticas como la gestión de la información, de los documentos y del conocimiento.

A continuación se presenta un esbozo de los fundamentos conceptuales de estos tipos de gestión en los que se enmarca esta experiencia.

Aunque estos tres conceptos han sido definidos de modos diversos y aún no hay un acuerdo universalmente aceptado desde la bibliotecología y las ciencias de la información sobre sus significados y límites, se asumen unas definiciones básicas y unas perspectivas orientadoras con el fin de explicar las experiencias vividas con respecto a estos tres procesos de gestión.

Más que en la conceptualización, se piensa en cómo estas

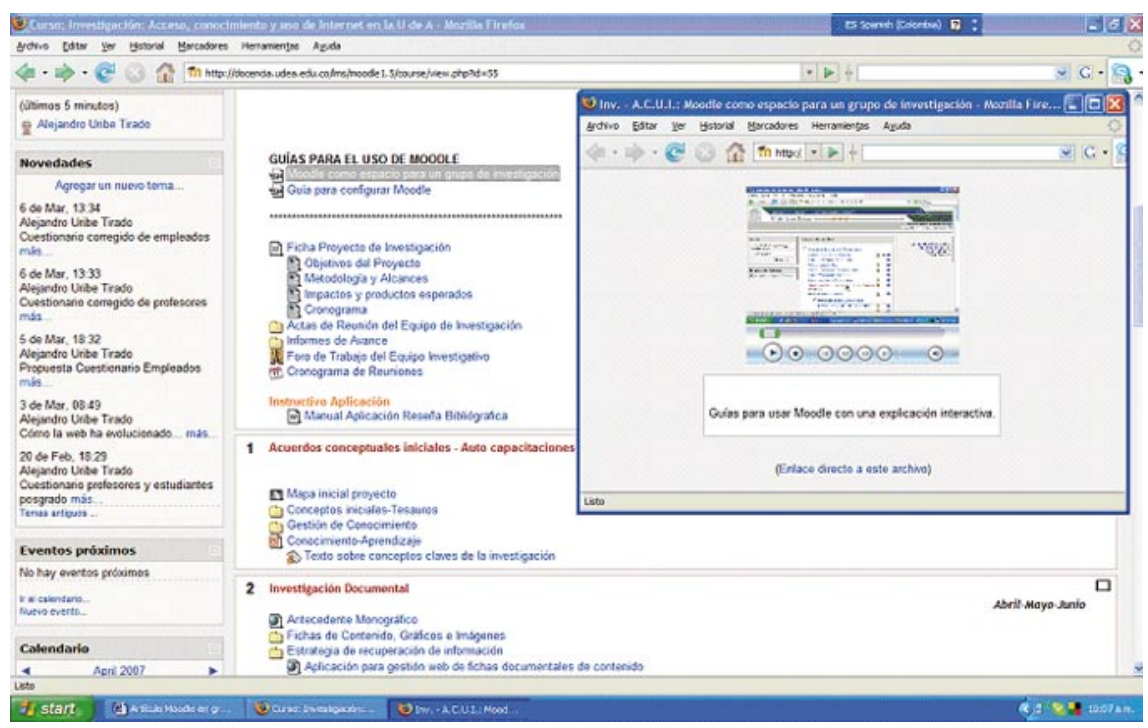

actividades se desarrollaron en la práctica de la investigación y en sus finalidades. Así, según FernándezMarcial, "el fin último de la gestión del conocimiento es permitir que éste sea compartido para ser utilizado, y la mejor forma de hacer esto es exteriorizándolo, plasmándolo en un medio que lo haga accesible a sus usuarios. Esto es, creando documentos, pues la relación entre información y/o conocimiento en un soporte es igual al concepto $d o$ cumento. Luego, también, es clave en la gestión del conocimiento la gestión documental" (FernándezMarcial, p. 59).

Según esta misma autora, una de las visiones que predomina de la gestión del conocimiento con relación a la gestión de la información es la de Nonaka y Takeuchi, según la cual aquella centra su labor en la gestión del conocimiento tácito, dejando para la gestión de la información todo lo relacionado con el conocimiento explícito (Fernández-Marcial, p. 58).

Los softwares de trabajo colaborativo permiten la conversión del conocimiento de tácito (manifestado en información que es expresada generalmente a través del discurso oral o informal, en reuniones o foros), a explícito (registrado en aplicaciones como foros, wikis, etc.). Este conocimiento convertido en documentos es objeto tanto de la gestión de la información (en el sentido de recuperación, uso y comunicación), como de la gestión documental (desde la perspectiva de su tratamiento propiamente documental).

\section{"Las plataformas de trabajo colaborativo permiten convertir el conocimiento de tácito a explícito"}

Figura 2. Esquema del módulo "Guía para el uso de Moodle" 
En esta experiencia se gestionó la información informal que circula en un grupo de investigación (diálogos, reuniones de trabajo, comunicaciones...) para que fuera plasmada en documentos para su posterior almacenamiento, recuperación y difusión. Dentro de este punto ubicamos también la producción de información documental de cualquier tipo y soporte (actas, informes, registro de bibliografía proporcionada por los miembros del grupo, y cualquier otra que se genere como producto de la comunicación durante la actividad investigadora y administrativa). Todo este trabajo es recogido y compartido a través de Moodle.

Como resultado se produce una serie de documentos que pasan a ser objeto del tratamiento documental propiamente dicho, es decir, de la gestión documental, sea a través de un programa estructurado para ello (como es el caso de uno de los grupos, que realiza tratamiento documental a toda esta producción), o de modo no automatizado o formalizado (labor que realiza otro grupo).

La gestión de conocimiento evidencia un requisito fundamental: la disposición a compartir, a recibir y divulgar experiencias, valores, información contextual, etc.; y es en ello que Moodle resulta de utilidad. En el mismo contexto de la web 2.0, estamos en una segunda generación en la gestión del conocimiento organizacional, que debe apoyarse de la gestión de conocimiento en red, tal como la define Luis-Ángel Fernández-Hermana: "Es un conjunto de procesos que permiten el trabajo colectivo en red y la generación de conocimiento social. O sea, creado por grupos o comunidades virtuales que se guarda en archivos que son transparentes para el acceso, tanto de esos grupos como de cualquier otro tipo de redes que lo necesiten (...). La información estratégica para nuestras organizaciones no está únicamente localizada entre sus paredes, en las cabezas de sus empleados o en su experiencia acumulada. Se encuentra también dispersa en redes electrónicas abiertas. La oferta y demanda de información a través de internet está determinando, cada vez más, el sentido de la nueva organización de cara a los retos de la sociedad".

En definitiva, se busca generar, interiorizar y socializar el conocimiento para que a partir de éste se desarrollen nuevos datos, información y conocimiento (el ciclo del conocimiento) para la toma de decisiones, ya sea en el contexto académico y de investigación o en el productivo y empresarial, en un entorno físico o en red. Todo ello implica tener en cuenta la finalidad última de esta gestión, como señala Bernardo Pérez-Castaño: "proceso de identificar, adquirir, utilizar y crear, tanto datos como información y conocimientos, relevantes, externos e internos a la organización, para mejorar tanto la eficiencia como la eficacia de la empresa (u organización pública o privada, con o sin ánimo de lucro) con el fin de garantizar su permanencia en el escenario competitivo (social)".

\section{Experiencias y lecciones aprendidas}

La utilización de Moodle para gestionar información, documentos y conocimiento de dos proyectos de investigación se podría resumir, entre otras, en las siguientes lecciones:

- Si un proyecto de investigación realiza una adecuada gestión, los conocimientos generados, al ser explícitos (documentos físicos o electrónicos), se convierten en la base para iniciar o continuar futuros proyectos relacionados con la temática tratada, sin partir de cero, sin duplicar esfuerzos y recursos, lo cual es uno de los postulados de la investigación holística, tal y como señala Jacqueline Hurtado-Barrera: "el ciclo holístico es un modelo que integra, organiza y concatena los holotipos de investigación (niveles y tipos de investigación) como momentos de un proceso continuo y progresivo, en el cual lo que un investigador deja a un cierto nivel, otros investigadores lo retoman para hacer de cada conclusión un punto de partida" (Hurtado-Barrera, p. 14).

- Moodle permite a los centros de investigación hacer un seguimiento de su quehacer tanto desde una perspectiva externa de evaluación y control como de autoevaluación y autocontrol entre los integrantes del grupo.

- El conocimiento tácito que se va generando se manifiesta en las discusiones (interacciones) que el grupo lleva a cabo durante el proceso de investigación, por lo que es una labor clave propiciarlo, ya sea en lo físico (actas de reunión) como en lo virtual (foros, chat, wikis).

- La utilización de recursos multimedia facilita el trabajo y el mismo proceso de aprendizaje que se da en una investigación. En estas experiencias se ha hecho uso de vídeos para trabajar colectivamente: "¿qué se entiende por determinado concepto?" para luego ser complementado por wikis, glosarios colaborativos o mapas conceptuales. A su vez, se ha trabajado la parte

"Si un proyecto de investigación realiza una adecuada gestión, los conocimientos generados, al ser explícitos (documentos físicos o electrónicos), se convierten en la base de partida de futuros proyectos" 


\section{"Tener un proyecto de investigación en una plataforma tecnológica de este tipo permite a los grupos de investigación hacer un seguimiento de su quehacer"}

de recolección y análisis de información apoyándose en grabaciones digitales de los entrevistados (en los aspectos cualitativos) y el análisis colectivo de la aplicación de este método contrastándolo con las fuentes primarias de las investigaciones.
- En Moodle se han integrado programas de manejo de citas, análisis de lenguaje y estadísticas (software Atlas Ti). Unas fichas online permiten un manejo bibliográfico colaborativo y de respeto a las fuentes originales (derechos de autor). A medida que cada investigador va en-

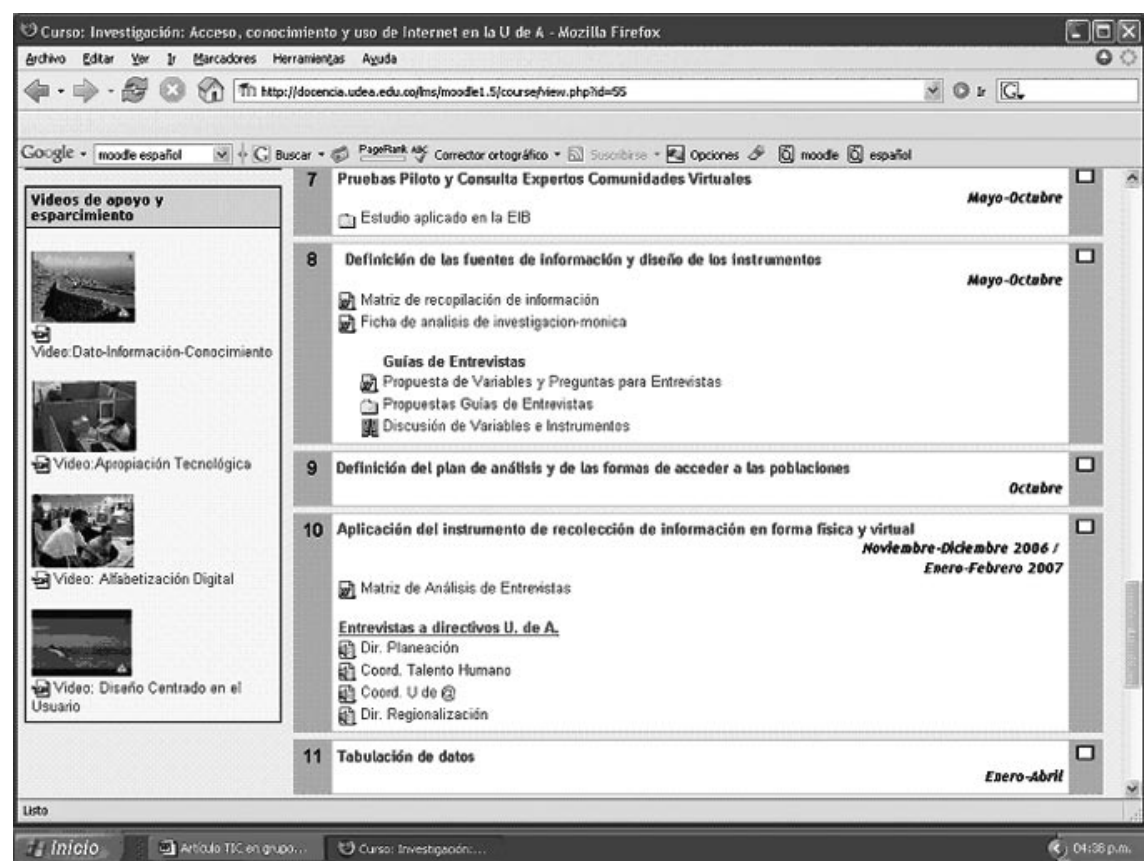

Figura 3. Muestra de los recursos multimedia usados por el proyecto de investigación de Moodle

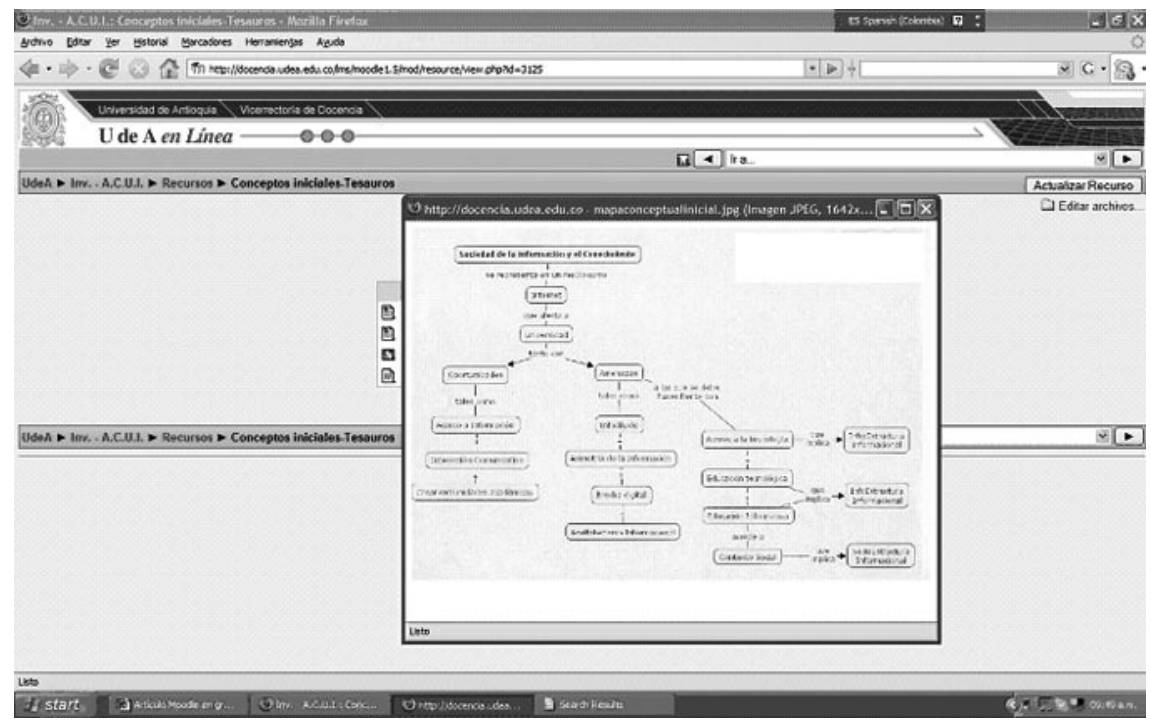

Figura 4. Integración y presentación de un mapa conceptual de la investigación contrando y analizando textos útiles para el marco teórico-conceptual y referencial de cada investigación, ingresa los datos clave de los mismos en formularios, creando así fichas bibliográficas. Eventualmente, a las mismas se pueden anexar imágenes y gráficos -como ficheros independientes- elaborados por el investigador al trabajar con la publicación.

- En el uso de Moodle se asumió la actitud proactiva y creativa de aprovechar los recursos tecnológicos existentes en las universidades y así lograr, como indica José Silvio en una de sus 10 reglas para la implementación adecuada de tecnologías de la información y la comunicación en las universidades, "superar y saber administrar las limitaciones (...) debido a una o varias de las siguientes situaciones: insuficiencia; inadecuación; utilización insuficiente; utilización inadecuada y alto costo".

- Se ha valorado la experiencia como una oportunidad excelente para la comunicación, para la escritura permanente sobre los hallazgos, reflexiones y elaboraciones sobre las temáticas, y para el intercambio efectivo de documentos. Sin embargo se ha resaltado la existencia de una brecha en el manejo de herramientas tecnológicas entre los investigadores, alguno de los cuales tardó en asimilarlas. Por tanto debe tenerse muy en cuenta la alfabetización digital e informacional que se requiere, pues no todos los investigadores tienen los mismos niveles ni provienen de profesiones o áreas temáticas familiarizadas con esta tecnología. Superada esta etapa se logra llegar a la utilización permanente y hasta a poder realizar una evaluación crítica del uso.

- La utilización de Moodle permite la fácil integración posterior de los resultados de los proyectos en la docencia, la investigación y la extensión en el ámbito universitario.

- Y finalmente, el trabajo del profesional de la información en 
los grupos de investigación pasa de ser un pasivo receptor de documentos creados, a un propiciador de su creación (o incluso ser su autor). El documentalista coadyuva en la elaboración de documentos colectivos, en la difusión de información ya generada por los miembros del grupo, en la transferencia eficaz de bibliografía, en el establecimiento de canales de comunicación, etc.

\section{Conclusión}

El principal resultado que deja esta experiencia es que en una investigación, desde la perspectiva de la gestión de información, documental y del conocimiento, es tan o más valioso el proceso que sus resultados, lo tácito que lo explícito. Por tanto, los grupos de investigación deben procurar la generación de estrategias (apoyados por profesionales de la información) para lograr esa complementariedad entre proceso y resultados. Para ello, las herramientas colaborativas y concretamente las plataformas que integran varias de ellas, como Moodle, son una alternativa muy valiosa, máxime en el caso de las universidades, donde es frecuente tener sistemas de administración de aprendizaje $(L M S)$ que se pueden adoptar para usos investigadores sin implicar inversiones adicionales.

A su vez, concluimos que la visibilidad investigadora está no sólo en las publicaciones formales (donde aparecen los resultados) sino también en los espacios virtuales que pueden dar cuenta del trabajo que se viene realizando (procesos) para compartirlo con pares de diferentes lugares del mundo. En nues- tro caso, brindamos la posibilidad de acceder (previa solicitud, mientras termina el proceso de investigación, y posteriormente de forma abierta) a los proyectos comentados en este trabajo:

http://docencia.udea.edu. co/lms/moodle1.5/course/view. php?id=55

http://docencia.udea.edu. co/lms/moodle 1.5/course/view. php?id=160

\section{Notas}

1. Se pensó en Joomla (aunque también se mencionaron Nuki, Spip, Plone o Drupal); o la implementación sólo de un wiki a través de un servidor web conectado a una intranet. Sin embargo, los wikis (mediawiki, dokuwiki, etc.), no permitían otras opciones de gestión para los grupos diferentes a la escritura colaborativa.

2. Moodle es un sistema de gestión de cursos libre (course management system) que ayuda a los educadores a crear comunidades de aprendizaje en línea.

http://es.wikipedia.org/wiki/Moodle

Es un paquete de software para la creación de cursos y sitios web, diseñado para dar soporte a un marco de educación social constructivista. http://docs.moodle.org/es/Acerca_de_Moodle

3. Para conocer otras experiencias del uso de Moodle para procesos de investigación se puede consultar este foro de su sitio oficial:

http://moodle.org/mod/forum/discuss. php?d=44397

4. Para analizar otras plataformas se puede consultar:

http://www.edutools.org/summative/index. jsp? $p j=8$

Si se desea tener acceso a estudios que ubican a Moodle como la plataforma seleccionada que daba mejor satisfacción al usuario en pequeñas y medianas empresas, y la segunda a nivel académico compitiendo con los grandes desarrollos comerciales:

http://www.elearningguild.com/pbuild/linkbuilder.cfm? selection $=$ doc. 1371

5. Según explican los encargados de tecnologías de la información para la docencia de la Universidad de Antioquia, "después del análisis técnico y pedagógico de las plataformas más utilizadas en la actualidad, el resultado fue la implementación de Moodle, por el modelo pedagógico que inspiró su creación que es constructivista y enfatiza en las actividades y en la participación de los estudiantes, además de contar con una comunidad académica conformada por importantes universidades de diversas partes del mundo, encargada de su desarrollo como un producto de software libre". Moodle se presentó entonces como una excelente opción, pues posibilita el acceso a contenidos y a la realización de actividades de interacción e interactividad gracias a las herramientas que la conforman: foros, gestión de contenido (recursos), cuestionarios con distintos tipos de preguntas, blogs, wikis, base de datos de actividades, encuestas, chat, glosarios, evaluación por pares.

Universidad de Antioquia. Portal Aprende en Línea. Curso Moodle para docentes:

http://aprendeenlinea.udea.edu.co/lms/moodle/ $\mathrm{mod} /$ resource/view.php?id $=26506$

6. Las herramientas colaborativas que generalmente han sido integradas por diferentes plataformas $L M S$, como Moodle, y más recientemente por $C M S$, para soportar procesos educativos (o de investigación como en nuestro caso) podemos clasificarlas de la siguiente manera:

\section{- Comunicación electrónica}

Enviar mensajes, archivos, datos o documentos entre personas y compartir información (colaboración asíncrona), como por ejemplo: correo electrónico, correo de voz, publicación en web;

- Conferencia

Para compartir información, de forma interactiva (colaboración síncrona), como por ejemplo:

conferencia de datos: PCs en red que comparten un espacio de presentación compartido y que cada usuario puede modificar;

- conferencias de voz: teléfonos que permiten interactuar a los participantes;

- conferencias de audio o de vídeo: PCs en red que comparten señales de audio o vídeo, salas de chat o mensajería instantánea, una plataforma de discusión para el intercambio inmediato de mensajes;

reuniones: sistema de conferencias integrado en una sala;

- Gestión colaborativa

Actividades del grupo, como por ejemplo:

calendarios electrónicos: para acordar fechas de eventos y enviar automáticamente notificaciones y recordatorios a los participantes;

sistemas de gestión de proyectos: organizar y hacer seguimiento de las acciones en un proyecto hasta que se finaliza;

sistemas de control de flujo de actividad: gestionar tareas y documentos en un proceso organizado de forma estructurada (burocracia);

sistemas de gestión del conocimiento: recoger, organizar, gestionar y compartir varios tipos de información;

- sistemas de soporte a redes sociales: organizar las relaciones de colectivos".

http://es.wikipedia.org/wiki/Groupware

\section{Bibliografía}

Fernández-Hermana, Luis-Ángel. Gestión de conocimiento en red. http://www.lafh.info

Fernández-Marcial, Viviana. "Gestión del conocimiento versus gestión de la información”. En: Investigación bibliotecológica, 2006, juliodiciembre, v. 20, n. 41, pp. 44-62.

García-Jiménez, Antonio. Organización y gestión del conocimiento en la comunicación. España: Trea, 2002. Isbn 8497040465.

\section{"Concluimos que la visibilidad investigadora está no sólo en las publicaciones formales sino también en los espacios virtuales que pueden dar cuenta del trabajo que se viene realizando"}


Hurtado-Barrera, Jacqueline. Metodología de la investigación holística. Caracas: Sypal-IUTC, 2000, 656 p. ISBN 9806306066

Izunza, Víctor. Citado por A3.Net. Definiciones de gestión de conocimiento. Consultado en: 1002-07.

http://www.a3net.net/es/gescon/definiciones.htm

Melo, Alexander. "Programas de gestión documental en el marco de las nuevas tecnologías. Consultado en: 21-02-07.

http://www.archivogeneral.gov.co/version $2 / \mathrm{htm} /$ tablas/sem/gesdocu.htm

Pérez-Castaño, Bernardo. "Estrategias de competitividad basadas en la gestión del conocimiento para pymes manufactureras de Cali" (Tesis doctoral). UPV Citado por A3.Net. Definiciones de gestión de conocimiento. Consultado en: 1002-07.

http://www.a3net.net/es/gescon/definiciones.htm

Ponjuán-Dante, Gloria. "Gestión documental, gestión de información y gestión del conocimiento: evolución y sinergias. Comunicación preliminar". En: Ciencias de la información, 2005, diciembre, v. 36, n. 03, pp. 67-71.

Ponjuán-Dante, Gloria. Gestión de información: dimensiones e implementación para el éxito organizacional. Argentina: Nuevo Paradigma, 2004, 208 p. Isbn 9879653661.

Silvio, José. “CCómo transformar la educación superior con la tecnología digital?". En: Martínez-Sánchez, Francisco; Prendes-Espinosa,

María-Paz (coord.). Nuevas tecnologías y edu- cación. Madrid: Pearson Pretince Hall, 2004, p. 93-112. Isbn 84-205-4162-1

Alejandro-Uribe Tirado, LilianaMaría Melgar-Estrada, Jaime-Alberto Bornacelly-Castro, Escuela Interamericana de Bibliotecología de la Universidad de Antioquia, Medellín, Colombia.

auribe@bibliotecologia.udea.edu. co

lilimelgar@gmail.com

jbornacelly@gmail.com

\title{
E- LIS E-prints in Library and Information Science eprints.rclis.org

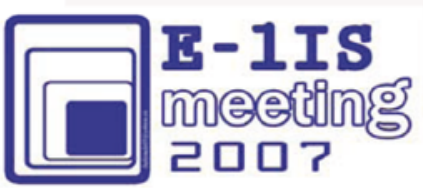

\section{E-LIS Meeting 2007: enhancing the access to LIS literature}

\author{
Valencia, Spain, $13^{\text {th }}-14^{\text {th }}$ November 2007
}

E-LIS is an OAI compliant archive on Library and Information Science since 2003

http://eprints.rclis.org

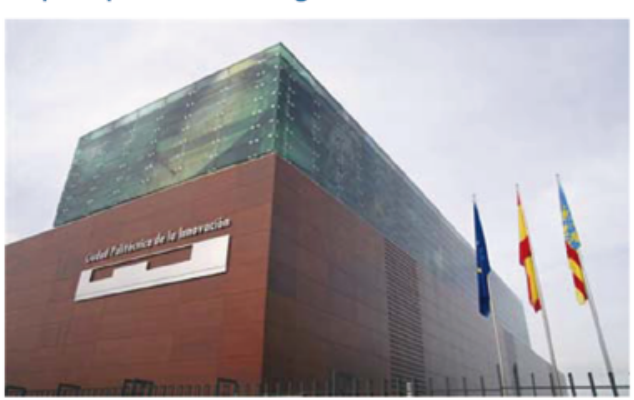

The E-LIS Meeting will be a good platform to disseminate to the professionals and to the society the benefits of the Open Access movement.

The meeting is addressed to researchers, librarians, policy makers, academic staff, information managers..., and all people related to science and technology.

\section{Venue}

Sala de Juntas - Fundación INNOVA, Ciudad Politécnica de la Innovación. Edif. 8E (Red Cube, 3rd floor).

Univ. Politécnica de Valencia, Camino de Vera s/n, 46022 Valencia.
Local Committee:

Fernanda Peset (UPV-DCADHA) Antonia Ferrer (UPV-DCADHA) Imma Subirats (FAO)

Nuria Lloret (UPV-DCADHA)

Antonio Hernández (UC3M)

Lourdes Palop (UPV-Library)

Elea Giménez (CINDOC-CSIC)
Tomàs Baiget (IDESCAT)

Scientific Committee:

Miguel Villamón (U Valencia) José Devis (UValencia) José Manuel Barrueco (UValencia) Antonella Di Robbio (U Padova) Thomas Krichel (U Long Island) Susanna Mornati (CILEA) José Antonio Ontalba (UPV-DCADHA) Josep Manuel Rodríguez Gairín (UB)

More information and registration: http://personales.upv.es/mpesetm/elis/ elis@upvnet.upv.es

\section{Speakers include:}

Josef Herget, Fac. for Info. Sci., Chur, CH Tim Brody, Univ. of Southampton, UK Paula Sequeiros, Inst. de Sociologia, Portugal Antoaneta Dimitrova, Techn. Univ. of Sofia Bozena B.-Michalska, Electronic Library, PL Danica Radovanovic, Nat. Library, Serbia Dennis Nicholson, Univ. of Strathclyde, UK
2. GENERALITAT VALENCIANA

\section{CONSELLERIA D'EMPRESA, UNIVERSITAT I CIÈNCIA} D.Gral. d'Investigació i Transferència Tecnológica

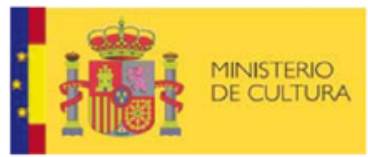

UNIVERSIDAD

POLITECNICA

DE VALENCIA
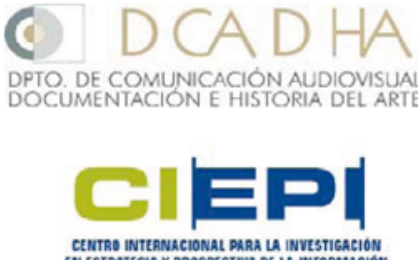

El profesional de la información

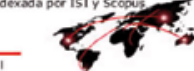

